\title{
Pupillographic findings in neglect
}

\author{
Manho Kim, Ronald L Schwartz, Kenneth M Heilman
}

\begin{abstract}
Objectives-Unilateral sensory neglect has been attributed to various defects, including a hemispatial attention-arousal deficit. However, support for this hypothesis has only been indirect. Therefore, the purpose of this study was to further test the hemispatial attentional-arousal hypothesis by measuring pupillary response as an index of arousal.

Methods-There were two experimental subjects with neglect and six matched controls. Stimuli (Arabic numbers) were presented on the right, left, and centre of a screen. The subjects were asked to look at the number in the centre, on the right, or left of the screen while their pupil diameter was measured.

Results-Unlike the control subjects, the subjects with neglect, who were aware of the left sided stimuli, did not show a pupillary dilatation when they looked at the stimulus on the left.

Conclusions-Although this study provides support for the hemispatial attention-arousal hypotheses of neglect, it does not preclude the possibility that other mechanisms may also be important. (F Neurol Neurosurg Psychiatry 1999;67:82-85)
\end{abstract}

Neurology, University of Florida College of Medicine, and the

Neurology Service, Department of Veterans Affairs

Medical Center, Gainesville, FL, USA

M Kim

K M Heilman

Department of Neurology, University of Florida at

Jacksonville, Jacksonville, FL, USA

R L Schwartz

Correspondence to: Dr Kenneth M Heilman, Box 100236, University of

Florida, Gainesville, FL

32610-0236, USA.

Telephone 0013523761611 ext 6058; or 001352392

3491; fax 001352392 6893;

email Heilman.@,

medicine.ufl.edu

Received 27 January 1998 and in final form

15 January 1999

Accepted 28 January 1999 jured left hemisphere. ${ }^{910}$ Based on these studies, if stimuli were projected solely to the lesioned hemisphere, one would expect a reduced arousal response, and this has already been demonstrated by Valler and his coworkers. ${ }^{11}$ However, these physiological studies do not provide evidence that stimuli that project to both hemispheres but are situated in contralesional body-head hemispace are determined by the brain to be less salient than stimuli in ipsilesional space.

The purpose of this study is to further test the hemispatial attentional-arousal hypothesis of neglect. Pupillary dilatation may be used as a behavioural index of stimulus-induced arousal. ${ }^{12}$ By using pupillary response as an index of stimulus-induced arousal, we attempted to learn if there is a hemispatial attention-arousal asymmetry associated with neglect.

\section{Methods}

SUBJECTS

There were two experimental subjects with neglect and six age and sex matched controls. The first experimental subject was 77 years old and the second 58. Both were men and right handed. Both were being treated for hypertension and came to the hospital for a left hemiparesis. On examination both were alert and fully oriented. Subject 1 had a right upper quadrantanopsia, Subject 2 had normal visual fields, and both showed a normal light reflex. Both demonstrated hemispatial neglect on line bisection (subject 1: $10.3(7.2) \mu \mathrm{m}$, subject 2 : $39.3(27.9) \mu \mathrm{m})$ and cancellation tasks. Both also had left sided visual and tactile extinction, a left hemiparesis with signs of left corticospinal injury such as increased reflexes, and extensor plantar responses. Brain CT on subject 1 showed a right hemispheric parietaloccipital haemorrhage. Subject 2 had a large infarction in a middle cerebral artery distribution that injured the frontal, temporal, and parietal lobes.

Six right handed men with a mean age of 64.2 (SD 14.9) years served as control subjects. All were without history of neurological or psychiatric diseases and were also not taking any medication that could influence the pupillary responses. We obtained informed consent from all subjects.

\section{APPARATUS}

We used an infrared eye monitoring instrument (Optiscan, Stoelting, Chicago, IL, USA) that has a sensitive resolution power better than 


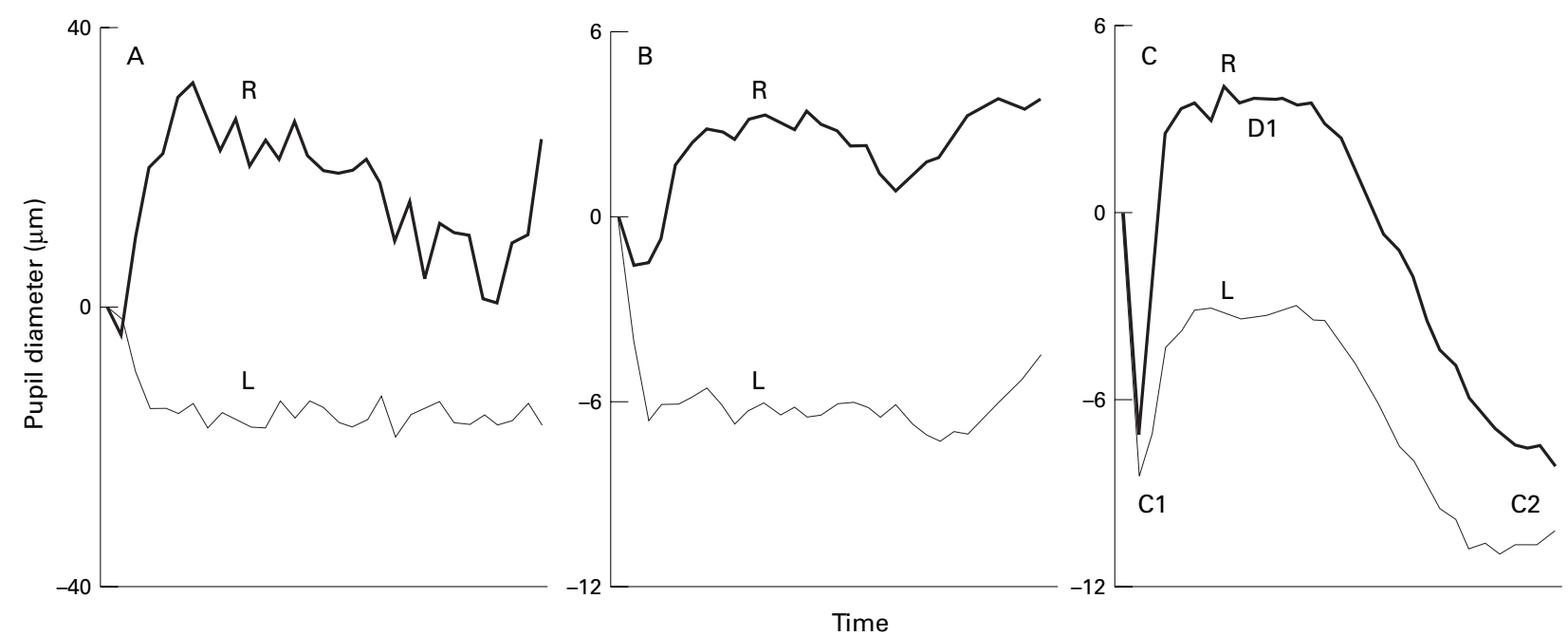

Averaged pupil responses for subjects 1 (A) and 2 (B) with neglect and six control subjects (C). $R=$ gaze to right; L=gaze to left; C1=first maximal constriction; $D 1=$ first maximal dilatation; $C 2=$ second constriction.

$0.1^{\circ}$ of visual angle and $0.02 \mathrm{~mm}$ of pupil movement and size. This apparatus monitors and collects data on both the size and position of the left eye 30 times/s enabling us to record rapid and small pupillary changes.

A computer monitor was placed so that the $28 \times 30 \mathrm{~cm}$ screen was $50 \mathrm{~cm}$ from the subjects' eyes. We enclosed the screen and testing space with black wooden panels $(80 \mathrm{~cm} \times 90 \mathrm{~cm} \times 70$ $\mathrm{cm})$ to control external illumination and reflections. During the testing procedure, the room lights were turned off and the computer screen's brightness was minimised.

\section{PROCEDURES}

Three $2 \times 2 \mathrm{~cm}$ sized white stimuli were projected onto the dark black screen: an Arabic number " 5 " in the centre of the screen, an Arabic " 4 " that was $9 \mathrm{~cm}$ to the left, and an Arabic " 6 " that was $9 \mathrm{~cm}$ to the right of the centre. The visual angle from the midline to lateralised stimuli was $10^{\circ}$.

During the experimental trials, the experimental and control subjects sat on an unmovable chair with their chin and forehead fixed by a chin brace and a forehead rest. The midsagittal plane of the body was aligned with the midsagittal plane of the head, and the number in the centre of the screen was aligned with the subject's midsagittal plane. The subject was then asked to look at the number " 5 " on the centre of the screen. While the subject was doing this, the investigator controlled the pupil image on the apparatus so that the pupil fell on the centre of the eye monitor. To make certain the experimental and control subjects could perceive the lateralised stimuli, the subject was first asked to look to the centre, then to the left and right of the centre, and to name the numbers that they saw. The experimental subjects with neglect could see the number " 4 " on the left, the number " 5 " in the centre, and number " 6 " on the right. The examiner also adjusted the infrared intensity to obtain a clear pupillary image. Then the examiner initiated the experimental trials by asking the patient to either look at the number " 4 " or " 6 ". The commands were presented in a pseudorandom order. After each of the trials, the examiner said the number " 5 " so that the subjects would return their eyes to the centre of the screen.

For each trial, data collection was started at least 1 second before the command and stopped at least 1 second after the patient made a saccade to the right or left. After the data were collected, it was transferred to an EXCEL (Microsoft, Cambridge, MA, USA) software program, which graphically displays pupillary size and eye position over time. If artifacts obscured these measurements, that trial was deleted.

We analysed pupil size for 1 second. This analysis started at the onset of the saccade to either the right or left hemispace. We defined the baseline pupil size as the size of the pupil when the subject looked at the central number " 5 ". Amplitudes of pupillary changes were measured from this baseline. We determined the time intervals from the initiation of saccade to peak points.

\section{Results}

The pupillary changes of the two experimental subjects with neglect were compared with those obtained from six normal subjects (figure). After removing those trials that contained artifacts such as blink, subject 1 looked rightward on 14 trials and leftward on 13 trials. Subject 2 looked rightward on 12 trials and leftward on 16 trials. For our normal subjects, there were 15.7 (SD 3.3) trials when these subjects looked rightward and 15.7 (SD 2.7) trials when they looked leftward.

Based on our studies of normal subjects' pupillary responses to stimuli on the right and left, we noted that these subjects had a common pupillographic pattern. At the beginning of the recording, there was a brief decrease of pupil size, which is termed the first maximal constriction (C1). The pupil then dilated. The dilatation lasted for $200-400 \mathrm{~ms}$ until the pupil reached its largest size, D1 (D1 is the first maximal dilatation). After D1, the pupil contracted again for another $400-700 \mathrm{~ms}$ and reached a second constriction point (C2). After 
Pupillographic data for controls and subjects 1 and 2 with neglect

\begin{tabular}{|c|c|c|c|c|c|c|}
\hline & \multicolumn{2}{|l|}{$C 1$} & \multicolumn{2}{|l|}{$D 1$} & \multicolumn{2}{|l|}{$C 2$} \\
\hline & Latency (ms) & Amplitude $(\mu m)$ & Latency (ms) & Amplitude $(\mu m)$ & Latency (ms) & Amplitude $(\mu m)$ \\
\hline \multicolumn{7}{|l|}{ Control* } \\
\hline Right & 29.2 to 42.6 & -57.7 to -6.4 & 217.1 to 349.5 & 27.3 to 57.8 & 866.9 to 975.7 & -126.4 to 10.6 \\
\hline Left & 36.0 to 86.2 & -85.3 to -35.3 & 158.1 to 519.1 & -39.6 to 6.8 & 881.0 to 961.6 & -121.3 to -32.9 \\
\hline R-L & 3.7 to 51.8 & 13.5 to 43.3 & -176.5 to 63.3 & 38.2 to 79.6 & -53.3 to 53.3 & -10.6 to 59.0 \\
\hline \multicolumn{7}{|c|}{ Subject 1} \\
\hline Right & 33.3 & -16.4 & 433.3 & 30.7 & 666.6 & 26.4 \\
\hline Left & 66.6 & -63.8 & - & - & - & - \\
\hline R-L & 33.3 & 47.4 & - & - & - & - \\
\hline \multicolumn{7}{|c|}{ Subject 2} \\
\hline Right & 33.3 & -40.0 & 233.3 & 320.0 & 899.9 & 5.0 \\
\hline Left & 99.9 & -145.0 & - & - & - & - \\
\hline R-L & 66.6 & 105.0 & - & - & - & - \\
\hline
\end{tabular}

${ }^{\star}$ Control data are at the $95 \%$ confidence interval.- = Unable to observe or define; $\mathrm{R}-\mathrm{L}=$ right - left difference.

reaching the second contraction peak, the pupil again dilated. Further study to learn the possible causes for these responses showed that D1 is associated with attention-arousal response, whereas $\mathrm{C} 1$ is generated by saccade, and C2 seems to be related to habituation or ocular fixation. Control subjects have asymmetric patterns with more pupillary dilatation when subjects look and attend to the stimulus on the right than when they look towards the left. ${ }^{13}$ Our control procedure showed that the observed asymmetries could not be attributed to accommodation, asymmetric illumination, or other stimulus parameters. These asymmetries, especially the D1 asymmetry, are related to the attention-arousal hypothesis that the stimuli in right hemispace may induce bilateral hemispheric activation and this may lead to greater arousal than attending to the stimuli in left hemispace.

The experimental subjects had an initial pupillary constriction (C1) on both the right and left gaze, as did the controls. The amplitudes and latencies of these responses are recorded in the table. The value of the right-left C1 interval difference for subject 1 was $33.3 \mathrm{~ms}, 66.6 \mathrm{~ms}$ for subject 2 , and 27.8 ms for control subjects, with $95 \%$ confidence intervals from $3.7 \mathrm{~ms}$ to $51.8 \mathrm{~ms}$. The value of the right-left $\mathrm{C} 1$ amplitude difference was $47.4 \mu \mathrm{m}$ for subject $1,105.0 \mu \mathrm{m}$ for subject 2 , and $28.3 \mu \mathrm{m}$ for controls with $95 \%$ confidence intervals from $13.5 \mu \mathrm{m}$ to $43.0 \mu \mathrm{m}$. Therefore, both the experimental subjects had a greater right-left difference than did the controls.

When the subjects with neglect looked and attended to the stimulus on the right after the initial constriction, there was pupillary dilatation, D1. The amplitude and latency for this dilatation can be found in the table. Confidence intervals based on the normal subjects' performance are also in the table. However, when the two subjects looked to the stimulus on the left, we could not see pupillary dilatation.

Due to the left-sided D1 response failure, we could not evaluate right-left differences in our experimental subjects or compare their performance with those of the control subjects.

After pupillary dilatation there is a second constriction (C2). The amplitude and latency of $\mathrm{C} 2$ along with confidence intervals can also be found in the table. However, due to the left-sided D1 response failure, we could not clearly define a left $\mathrm{C} 2$ and could not evaluate the right-left $\mathrm{C} 2$ differences.

\section{Discussion}

Physiologically, arousal is defined as the excitability of the nervous system. ${ }^{14}$ An arousal response is often associated with increased sympathetic activity, and sympathetic activity induces pupillary dilatation, making the pupillographic method a means of measuring the state of arousal. There are several other psychophysiological means of measuring arousal. However, if an arousal response is brief or slight, it may be difficult to detect or measure using techniques such as heart rate or galvanic skin responses. Therefore, the pupillographic method can provide a sensitive measure for changes of arousal.

Several investigators have used psychophysiological methods to study arousal in patients with neglect. ${ }^{15-17}$ Although these studies demonstrate that patients with right temporoparietal lesions and neglect have an overall reduction in their level of arousal, they did not attempt to learn if looking to stimuli that projects to both hemispheres, presented on the side of space contralateral to the lesion, induces a smaller arousal response than looking to stimuli presented to the side of space that is ipsilateral to the hemispheric lesion.

Normally, after the initial pupillary constriction (C1), the relevance of which is unknown, the pupils dilate (D1). Unlike controls, the experimental subjects, who had right hemispheric lesions, did not demonstrate a clear D1 response when looking at the stimulus presented in left (neglected) hemispace, but their pupils did dilate when they looked at the stimulus in right hemispace. Because these patients were able to move their eyes to the left side of space and read the number that appeared in the left side of space, this hemispatial arousal deficit cannot be explained by hemianopia or sensory defect. In addition, this defect seems to be hemispatial (to the left of the body and head midline) rather than retinotopic (in the left visual field). Hemispace in this study was defined by both the head and body. Because our experimental subjects' pupils did dilate when they looked to the right, the failure of the pupils to dilate when the subjects looked to the left cannot be explained by the previously described generalised arousal deficit associated with right hemispheric dysfunction. ${ }^{15-17}$ 
Severe inattention may produce unawareness. Although our subjects were aware of the left sided stimulus, they had reduced arousal when looking to the left. Had we increased attentional demands or used a vigilance paradigm, we may have noted hemispatial behavioural deficits. We studied one patient with a left hemispheric lesion who was without neglect, and this patient did not show the pupil asymmetries. However, we need a larger population of subjects with hemispheric lesions, with and without neglect, to learn if these pupillary asymmetries are associated with neglect or right hemispheric lesions independent of neglect. Lastly, although the pupillary asymmetries we found cannot be directly attributed to either an inability to disengage from ipsilesional stimuli or an ipsilesional spatial bias, the finding that patients with hemispatial neglect have a hemispatial attentionarousal deficit does not preclude the possibility that patients with neglect may also have these other deficits.

We thank Barbara Haws for technical support, Gregory Crucian, Anna Barrett, and Jeff Anderson for useful comments, Anne Hines for manuscript preparation, and Janet Wootten for editing the manuscript. This work was supported by the Medical Reqsearch Service of the Department of Veterans Affairs.

1 Heilman KM, Watson RT, Valenstein E. Neglect and related disorders. In: Heilman KM, Valenstein E, eds. Clinical neuropsychology. New York: Oxford University Press; 1993:279-336.
2 Heilman KM. Neglect and related disorders. In: Heilman KM, Valenstein E, eds. Clinical neuropsychology. New York: KM, Valenstein E, eds. Clinical neuropsychol
Oxford University Press; 1979:268-307.

3 Watson RT, Valenstein E, Heilman KM. Thalamic neglect; the possible role of the medial thalamus and nucleus reticularis thalami in behavior. Arch Neurol 1981;38:501-7.

4 Mesulam MM. A cortical network for directed attention and unilateral neglect. Ann Neurol 1981;10:309-25.

5 Kinsbourne M. Direction of gaze and distribution of cerebral thought processes. Neuropsychologia 1974;12:270-

6 Posner MI, Walker J, Friedrich FJ, et al. Effects of parietal lobe injury on covert orienting of visual attention. $\mathcal{F}$ Neurosci $1984 ; 4: 163-87$.

7 Mark VW, Kooistra CA, Heilman KM. Hemispatial neglect affected by non-neglected stimuli. Neurology 1988;38: $1207-11$

8 Riddoch MJ, Humphreys G. The effect of cuing on unilateral neglect. Neuropsychologia 1983;21:589-99.

9 Watson RT, Miller BD, Heilman KM. Evoked potential in neglect. Arch Neurol 1977;34:224-7.

10 Fiorelli M, Blin J, Bakchine S, et al. PET studies of cortical diaschisic in patients with motor hemi-neglect. $\mathcal{F}$ Neurol Sci 1991;104:135-42.

11 Vallar G, Bottini G, Sterzi R, et al. Hemianesthesia, sensory neglect, and defective access to conscious experience. Neurology 1991;41:650-2.

12 Bradshaw JL. Pupil size as a measure of arousal during information processing. Nature 1967;216:515-16.

$13 \mathrm{Kim} \mathrm{M}$, Barrett A, Heilman KM. Lateral asymmetries of kim M, Barrett A, Heilman KM. Lateral a

14 Wurtz RH, Goldberg ME, Robinson DL. Behavioural modulation of visual responses in the monkey: stimulus selection for attention and movement. In: Sprague JM, Epstein AN, eds. Progress in psychobiology and physiological psychology. New York: Academic, 1980:9:43-83.

15 Heilman KM, Schwartz HD, Watson RT. Hypoarousal in patients with neglect syndrome and emotional indifference. Neurology 1978;28:229-32.

16 Schrandt NJ, Tranel D, Damasio H. The effects of total cerebral lesions on skin conductance response to signal stimuli. Neurology 1989;39(suppl 1):223.

17 Yokoyama K, Jennings R, Ackles P, et al. Lack of heart rate changes during an attention demanding task after right hemisphere lesions. Neurology 1987;37:624-30. 